\title{
Transplantation after Blunt Trauma to the Liver: A Valuable Option OR JUST A "WASTE OF ORGANS"?
}

\author{
M. Heuer ${ }^{1}$, G. M. Kaiser ${ }^{1}$, S. Lendemans ${ }^{2}$, S. Vernadakis ${ }^{1}$, J. W. Treckmann ${ }^{1}$, A. Paul ${ }^{1}$ \\ ${ }^{1}$ Department of General-, Visceral- and Transplantation Surgery, ${ }^{2}$ Department of Trauma Surgery, \\ University Hospital of Essen, Germany
}

\begin{abstract}
Objective: Liver injury due to trauma is a rare indication for transplantation. The main indications in such cases were uncontrollable bleeding and insufficient hepatic function. Because of poor results, liver transplantation in these patients is occasionally described as "waste of organs", however based on insufficient data. This study aims to report our experience and to critically question the indication of transplantation in these patients.

Methods: All liver transplantations at our institution were reviewed retrospectively. This covered 1,529 liver transplants between September 1987 and December 2008. Of them, 6 transplants were performed due to motor-vehicle accidents which caused uncontrollable acute liver trauma in 4 patients. The patients' peri-operative course, short- and long-term outcomes were analyzed.

Results: Five deceased-donor liver transplantations (4 full size, 1 split) and 1 living donor (right) transplantation were performed. The median GCS score was 9/15; the median MELD score was 15. Postoperative complications were observed in 3 patients, requiring re-operation in 2 . After a median (range) follow-up of
\end{abstract}

32.95 (10.3-55.6) months, 2 patients are alive and remain well on immunosuppression.

Conclusion: Liver transplantation in patients with otherwise surgically uncontrollable acute liver injury can be indicated as a life saving procedure and can be performed successfully in highly selected cases.

Key words: abdominal trauma, liver injury, liver transplantation, prognosis, "waste of organs"

\section{INTRODUCTION}

The liver is one of the most commonly injured organs of the abdomen and its trauma often causes bleeding. Therefore, almost $90 \%$ of the liver injuries originated by blunt trauma in polytraumatized patients in Europe. Of those, more than $90 \%$ are treated successfully in a conservative way. Merely $10 \%$ of these liver trauma patients - mostly of severity IV and $\mathrm{V}$ and with an increased mortality rate of $46 \%$ respectively $80 \%$ - receive surgical therapy, Table $1,[1-3]$.

The management of a liver injury aims to control hemorrhage, preserve sufficient hepatic function and prevent secondary complications. If an adequate con-

Table 1. American Association for the Surgery of Trauma (AAST) -scale and modified scale for classification of liver injuries.

\begin{tabular}{|c|c|c|c|}
\hline $\begin{array}{l}\text { AAST } \\
\text { Grade }\end{array}$ & Injury & Injury Description & $\begin{array}{l}\text { AIS-98* } \\
\text { Grade }\end{array}$ \\
\hline \multirow{2}{*}{ I } & hematoma & subcapsular, $<10 \%$ surface & 2 \\
\hline & laceration & capsular tear, $<1 \mathrm{~cm}$ parenchymal depth & 2 \\
\hline \multirow{2}{*}{ II } & hematoma & subcapsular, $10-50 \%$ surface; intraparenchymal hematoma, $<10 \mathrm{~cm}$ in diameter & 2 \\
\hline & laceration & capsular tear, $1-3 \mathrm{~cm}$ parenchymal depth, $<10 \mathrm{~cm}$ length & 2 \\
\hline \multirow{2}{*}{ III } & hematoma & subcapsular, $>50 \%$ surface; intraparenchymal hematoma, $>10 \mathrm{~cm}$ in diameter & 3 \\
\hline & laceration & $>3 \mathrm{~cm}$ parenchymal depth & 3 \\
\hline IV & laceration & parenchymal disruption involving $25-75 \%$ of hepatic lobe or $1-3$ segments & 4 \\
\hline \multirow{2}{*}{$\mathrm{V}$} & laceration & parenchymal disruption involving $>75 \%$ of hepatic lobe or $>3$ segments within a single lobe & 5 \\
\hline & vascular & hepatic venous injuries & 5 \\
\hline VI & vascular & hepatic avulsion & 6 \\
\hline
\end{tabular}

*Note-AIS-98 = Abbreviated Injury Scale, 1998 version. 


\begin{tabular}{|c|c|c|c|c|}
\hline 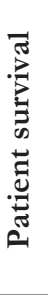 & 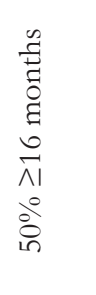 & 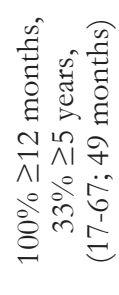 & 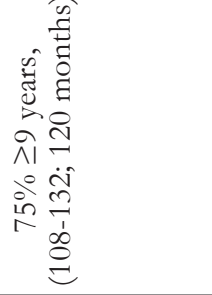 & 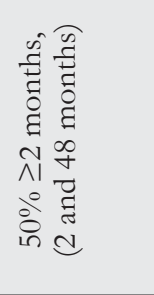 \\
\hline 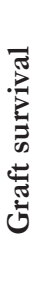 & & & 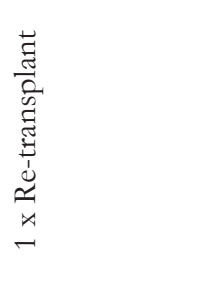 & 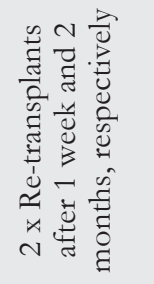 \\
\hline 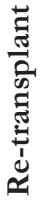 & & & $\overrightarrow{\|}$ & $\begin{array}{l}N \\
I \\
\simeq\end{array}$ \\
\hline 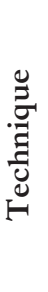 & 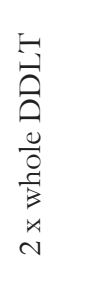 & 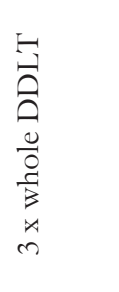 & 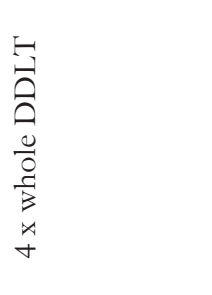 & 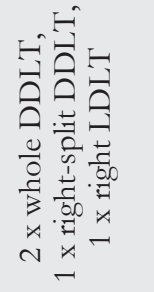 \\
\hline 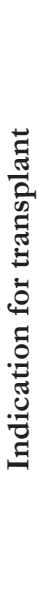 & 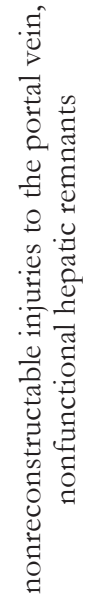 & 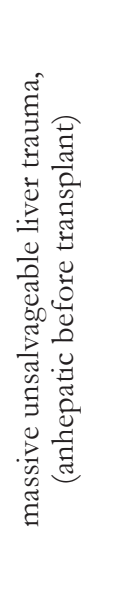 & 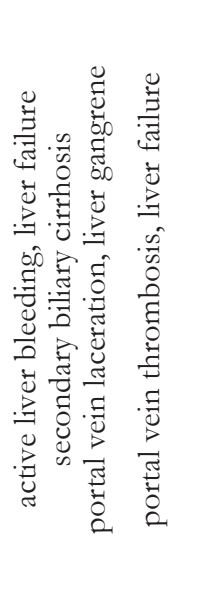 & 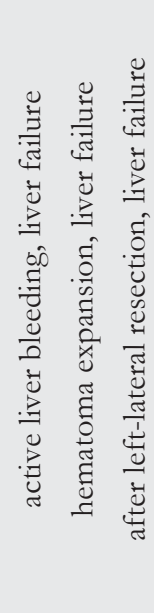 \\
\hline 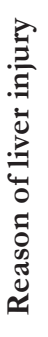 & 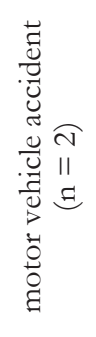 & 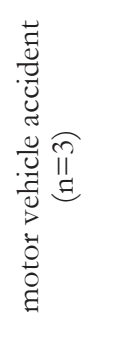 & 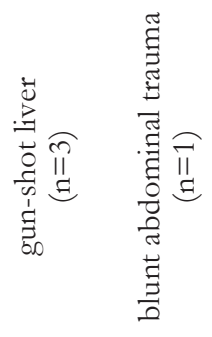 & 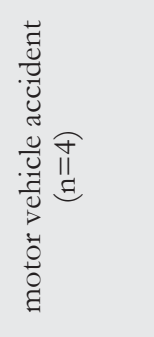 \\
\hline 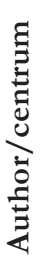 & 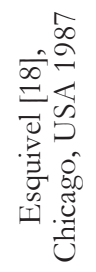 & 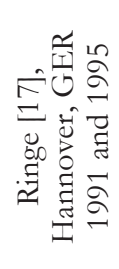 & 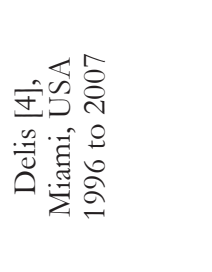 & 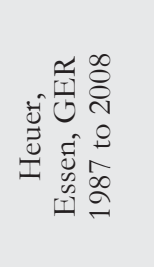 \\
\hline
\end{tabular}

trol of the bleeding cannot be achieved despite exhausting the current therapy options, the indication for liver transplant (LT) needs to be assessed critically in individual cases. These cases are extremely scarce in the clinical daily routine [4].

Nonetheless, LT are carried out due to acutely uncontrollable liver injuries in exceptional cases only. For this, indication is judged critically and discussed controversially due to usually existing secondary injuries, early septic complications, and poor general condition. Due to poor results, LT in these patients is occasionally described as "waste of organs", however based on insufficient data $[5,6]$.

Patients with subacute and chronic results of a liver injury need to be considered differently from the acute and due to their initial position very special group of surgically uncontrollable patients with liver trauma. However, they share the fact that also the indication for transplantation for instance in patients with "shock liver" in the context of polytrauma or with induced liver failure after a longer intensive therapy need to be measured $[7,8]$.

Our study was aimed to critically question the indication of LT on the basis of blunt and uncontrollable liver trauma; we therefore report our experience with 4 patients who all underwent LT due to accidentcaused uncontrollable acute liver trauma at our center along with a comparison and discussion of our results based on the current literature, Table 2.

\section{Methods}

From September 1987 to December 2008, our center performed 1,529 LT ( 6 traumatic and 1,523 others in 4 and 1,475 patients, respectively). Apart from transplant surgery, the clinic's second major focus is on hepatobiliary surgery. In this analysis the following eligibility criteria were used:

1. patients $\geq 18$ years;

2. trauma-caused blunt liver injury;

3. uncontrollable clinically situation without transplantation.

The transplantations conformed to the local ethical guidelines and followed the ethical guidelines of the 1975 Declaration of Helsinki. LT was indicated in cases of uncontrollable liver injuries. It was considered contraindicated in cases of irreversible cerebral damage (i.e. slight cerebral edema is not considered a contraindication), absence of uncontrolled extrahepatic infection (i.e. no SIRS), absence of uncontrolled multiple organ failure (MOF) (less than 3 organs including the liver).

In order to offer the best sized organ in a timely fashion, the following surgical procedures were considered for all recipients when available: deceased donor liver transplantation (DDLT) (full size and splitleft lateral, left, right, extended right) and living donor liver transplantation (LDLT) (left lateral, left, right).

The conservative management of our patients consisted of: a) causal therapy, b) intense monitoring of hemodynamic, respiratory, renal, neurological, infectious, hepatic and metabolic parameters, c) minimal 
handling and no sedation whenever possible, d) fluid restriction but enough fluid to assure cerebral perfusion, e) hypercaloric protein-free nutrition, f) intestinal sterilization with Neomycine and Lactulose, g) fresh frozen plasma in cases of coagulation disorder. All patients received immunosuppressive induction with Prednisolone. Maintenance immunosuppression consisted of a dual therapy with calcineurin inhibitors and Prednisolone post-transplant.

We monitored the peri-operative course of each patient and noted short-term and long-term outcomes. The end of follow-up for this study was the end of July 2009. Continuous variables are expressed as mean ( $\pm \mathrm{SD})$ or median (range).

\section{RESULTS}

\section{CASE 1}

A 38-year-old polytraumatized male presented with an acute abdomen after a motor vehicle accident. His GCS score was 9/15 upon admission. The patient was initially managed according the "advanced trauma life support" (ATLS) guidelines and shortly thereafter was transferred to the operation room, due to signs of active abdominal bleeding. During an exploratory laparotomy, a central rupture of the liver was documented, with active bleeding. A Pringle maneuver was initially used for approximately 20 minutes along with the attempt of getting the destroyed liver tissue free of blood with diffuse stitches. The abdomen was then packed. On the same, day the patient remained hemodynamic unstable with further bleeding from the liver injury requiring re-exploration. Due to the uncontrollable situation and without any chance for surgical repair of the vascular and parachymal rupture the patient was packed again and transferred to the ICU with a plan for transplant. Liver and renal functions deteriorated progressively, with persistent acidosis, desolate blood clotting and increasingly worse hemodynamic condition. The patient was listed for transplant with MELD 29 and underwent an orthotopic liver transplant at the same day. After transplantation he was in very bad hemodynamic condition ongoing; he developed sepsis at pod 11 and was specific antibiotic treated in the ICU. On the 17th post-operative day the patient died due to MOF.

\section{CASE 2}

Analogous case 1, a 44-year-old polytraumatized man with abdominal trauma due to a motor vehicle accident underwent emergency laparotomy because of signs of abdominal bleeding. Intra-operatively, a complete liver destruction left no option for any further surgery; splenectomy was performed due to laceration, American Association for the Surgery of Trauma (AATS) grade V. The patient was packed and transferred to our ICU. Furthermore, the patient was directly listed "high urgency" for transplant. Due to the expected waiting period at this point of time and at the request of his family, we began with emergent evaluation of a possible living donor. After a 4-day waiting period without applicable organ and with continuously deteriorating of the overall situation, he un- derwent a right LDLT by his brother. The postoperative course was totally uneventful. Fifty-five months after the operation the recipient and donor are alive and doing well.

\section{CASE 3}

3 A 50-year-old man developed a subcapsular hematoma mainly of the right liver lobe due to a blunt abdominal trauma caused by a motor vehicle accident. GCS was 15/15 upon admission. Because of hematoma expansion we performed a right hemihepatectomy. Postoperatively his liver and renal functions deteriorated rapidly. He presented with hepatic encephalopathy grade I. The cerebral CT scans showed minimal cerebral edema, and the EEG was normal. His INR was 1.16 , bilirubin $4.8 \mathrm{mg} / \mathrm{dl}$, and creatinine $1.15 \mathrm{mg} / \mathrm{dl}$. His MELD was 15 up to the time of listing. There was no hepato-renal syndrome, but he needed mechanical ventilation and pressor support. He underwent a full-size DDLT after just 2 days waiting time. Postoperatively, the graft demonstrated perfect primary function, and the patient recovered quickly. On the 17th day post-op though, he developed cholestasis due to ischemic-type biliary lesions type (ITBL) III. After interventional ERCP on post-op day 20 and ongoing cholestasis, a biliodigestive anastomosis was redone immediately, but he experienced cholestasis again with consequent graft loss. The patient was re-transplanted 2 months after the first transplant with a full size DDLT. Ten months after the retransplantation, the patient is alive and doing fine.

\section{CASE 4}

A 36-year-old female was admitted with accidentcaused blunt liver trauma. GCS was 3/15. She presented in hemorrhagic shock. After initial emergency room management she underwent laparotomy with resection of the completely destructed left-lateral liver lobe. Postoperatively she had a longer need for mechanical ventilation and pressor support. Liver and renal function, however, deteriorated progressively. A then developed CT scan of the abdomen generated, in combination with the lab values, the diagnosis of a shock liver. After 30 days of waiting time, she received a right split DDLT. The liver graft developed primary non function. On the 7th day post-op, the patient was re-transplanted with a full size DDLT. Then the liver graft had a good primary function, but unfortunately, she received therapeutic-resistant sepsis. Seven days after the second LT, the patient died due to MOF.

Six LT were performed in 4 patients with acute liver injury (2 patients were re-transplanted). In Table 3 , the demographics and the clinical presentation of these patients are reported. There were 3 men and 1 woman, ranging in age from 36 to 50 years (mean and median, 42 years and 41 years, respectively). All patients had uncontrollable liver injuries caused by motor vehicle accidents. After a median (range) follow-up of 32.95 months (10.3-55.6), 2 out of 4 patients are still alive. Half- and 4-year patient survival rates are 50\% and $25 \%$ with a corresponding graft survival of $25 \%$, respectively. 
Table 3. Demographics and clinical presentation of LT due to uncontrollable acute liver trauma in 4 patients at the University Hospital of Essen.

\begin{tabular}{|c|c|c|c|c|}
\hline Age (years) & $\begin{array}{l}\text { Median } \\
18-40 \\
41-50 \\
51-60\end{array}$ & & $\begin{array}{l}41 \\
2 \\
2 \\
0\end{array}$ & $(36-50)$ \\
\hline Gender & $\begin{array}{l}\text { Male } \\
\text { Female }\end{array}$ & & $\begin{array}{l}3 \\
1\end{array}$ & \\
\hline $\begin{array}{l}\text { Clinical } \\
\text { presentation }\end{array}$ & $\begin{array}{l}\text { HE grade } \\
\text { Jaundice } \\
\text { Ascites } \\
\text { Ventilation support } \\
\text { Pressor support } \\
\text { Haemodialysis }\end{array}$ & $\begin{array}{l}0 \\
\text { I } \\
\text { II } \\
\text { III } \\
\text { IV }\end{array}$ & $\begin{array}{l}3 \\
1 \\
0 \\
0 \\
0 \\
1 \\
1 \\
3 \\
3 \\
1\end{array}$ & $\begin{array}{l}25 \% \\
25 \% \\
75 \% \\
75 \% \\
25 \%\end{array}$ \\
\hline $\begin{array}{l}\text { Lab values; } \\
\text { median } \\
\text { (range) }\end{array}$ & $\begin{array}{l}\text { INR } \\
\text { Bilirubin }(\mathrm{mg} / \mathrm{dl}) \\
\text { Creatinine }(\mathrm{mg} / \mathrm{dl})\end{array}$ & & $\begin{array}{l}1,16 \\
4,8 \\
1,15\end{array}$ & $\begin{array}{l}(1,01-2,3) \\
(1-6,6) \\
(0,97-4,2)\end{array}$ \\
\hline MELD & $\begin{array}{l}\text { Mean } \\
\text { Median }\end{array}$ & & $\begin{array}{l}19,33 \\
15\end{array}$ & $\begin{array}{l} \pm 8,39 \\
(14-29)\end{array}$ \\
\hline
\end{tabular}

\section{Discussion}

Treatment of liver trauma has rapidly changed over the past decades. Thus, especially development of the intensive and emergency medicine as well as coagulation substitution reveal a more and more conservative therapy approach against the severity of the injury. To date, merely $10 \%$ of the liver trauma patients are surgically treated, $90 \%$ follow a conservative therapy regimen. In the process, the overall mortality of $60 \%$ could be reduced to about $6 \%$ over the past century [9-11].

However, in a few patients with liver injuries it may still occur that they cannot be treated adequately despite exploitation of all conventional surgical measures. Continuous non-controllable acute bleeding, non-reconstructible liver injuries, like e.g. injuries of the liver's veins or the bile duct system, and a liver insufficiency caused by trauma, e.g. shock liver, allow for the consideration of $\operatorname{LT}[12,13]$.

LT then remains the only available life-saving procedure for these patients. However, not all patients are suitable candidates for LT. Pre-transplant neurological status, severe sepsis, MOF, and accompanying severe injuries may all be contraindications to LT. Furthermore, there is a fundamental difference whether a patient is transplanted due to acute non-controllable liver injury or due to subacute (e.g. shock liver) respectively chronic (e.g. secondary biliary cirrhosis) liver mutation after occurred trauma. Ultimately, only a fraction of patients with uncontrollable liver trauma are deemed to be candidates for transplantation. Like those patients who die before LT, mortality after LT is usually secondary to hemodynamically instability, infections and MOF $[14,15]$.
The underlying severity of the injury and the occasionally life-threatening other injuries are reflected by the results in our patients who received a LT due to trauma all from a motor-vehicle accident. These patients differ fundamentally from the majority of our other liver transplant patients in the peri-operative prognosis. Based on our clinical experience, the most relevant preoperative prognostic factors negatively influencing the post-transplant outcome have been the hemodynamic, secondary injuries and the recipient age. There are diverging opinions about the role of the MELD score as a prognostic factor for the postoperative outcome in such cases.

The results following LT for uncontrollable traumatic liver injuries are substantially worse than those of LT for sub-acute/chronic and elective indications. In fact, the general patient survival rates are approximately $50-75 \%$. Unfortunately, the few reported cases in the current literature are quite inhomogeneous, reflecting different transplant eras, clinical experience, LT techniques/procedures, and clinical conditions of the patients prior to undergoing LT. In addition these case reports mostly outline the clinical course of liver transplant patients following trauma. While accurate comparison of the clinical presentation of patients across various case reports is not always possible, we can say, based on the available data in 3 case series, that the clinical conditions of our patients appear to be similar to those reported [16-18].

As shown in Table 2, Delis et al. also describe 4 patients with liver trauma in their work which are transplanted in the course of their disease. Nonuniform genesis of these patients are reflected in a range of relatively positive GCS scores. These may be explained by the fact that 3 of the above-mentioned patients had gun-shot liver injuries and hence no, as common in blunt liver injuries, large-area, complex liver injuries. Furthermore, one patient was transplanted after two years due to secondary biliary cirrhosis caused by trauma. This explains the fairly good results in this group with a patient survival rate of $75 \%$ after more than 9 years.

Altogether 3 patients with liver injuries due to car accidents, that were hepatectomized pre-operatively due to massive unsalvageable liver trauma, are described by Ringe et al. This quite more homogenous patient population is better comparable to our study and demonstrated a patient survival rate geared to our results. Thereby, Ringe postulates a bilateral approach in patients where no sufficient hemostasis after liver trau$\mathrm{ma}$ is achievable. After an indication for total hepatectomy depending on hemodynamic parameters, a than obligatory liver transplantation is carried out as soon as possible. In his works, however, also patients are described that could not be allocated with an adequate organ in time due to the present lack of donor organs.

Also comparable with our results are those published in the 1980ies by Esquivel et al. on 2 traffic accident victims with nonreconstructable injuries to the portal vein and following nonfunctional hepatic remnants. In literature, these are the first published cases of liver transplantations after liver trauma.

The majority of our patients demonstrated one or more of negative prognostic factors. This study covers 
all recorded liver transplantations for otherwise uncontrollable liver trauma due to motor-vehicle accidents at our hospital. These cases often had poor general prognoses. Despite the acute condition of our patients, our results, patient survival rate is $50 \%$ with a corresponding graft survival of $25 \%$, are among the first reports on survival rates in a homogenous series to date in the literature.

In summary, we largely agree with the aforementioned reports. The therapeutic option of liver transplantation also needs to be accessible for patients with liver injuries caused by trauma. However, not least due to the mentioned poor transplantation results in severely injured patients, indication for transplantation needs to be critically proposed by the attending surgeons. It is essential to sensibly and appropriately allocate the organs so that the shortage of donor organs is not further enlarged. In patients where no hemodynamic stabilization can be achieved despite exhaustion of all extensive care measures, transplantation should not be considered any further. Although, there is a fundamental difference regarding the timeframe after trauma during which patients are to be transplanted. It has shown, that especially patients with acute, non-controllable liver injuries as described by us have clinically changed for the worse rapidly after transplantation and have died in MOF. Therefore, we postulate that indication for transplantation in these patients may only be provided after critically reviewing every single case as not to "waste of organs".

Comflict of Interest: Nothing to declare.

\section{REFERENCES}

1. Heuer M, Taeger G, Kaiser GM, et al. Prognostic factors of liver injury in polytraumatic patients. Results from 895 severe abdominal trauma cases. J Gastrointestin Liver Dis 2009;18:197-203.

2. Kaiser GM, Kuehne CA, Paul A, et al. Nonoperative management of gunshot liver trauma in a severely injured patient. Injury Extra 2006;37:228-229.

3. Moore EE, Cogbill TH, Jurkovich GJ, Shackford SR, Malangoni MA, Champion HR. Organ injury scaling: spleen and liver (1994 revision) J Trauma 1995;38:323324.

4. Delis SG, Bakoyiannis A, Selvaggi G, Weppler D, Levi D, Tzakis AG. Liver transplantation for severe hepatic trauma: Experience from a single center. World J Gastroenterol 2009;15:1641-1644.

5. O'Grady JG, Alexander GJ, Hayllar KM, Williams R. Early indicators of prognosis in fulminant hepatic failure. Gastroenterology 1989;97:439-445.
6. Schnüriger B, Inderbitzin D, Schafer M, Kickuth R, Exadaktylos A, Candinas D. Concomitant injuries are an important determinant of outcome of high-grade blunt hepatic trauma. Br J Surg 2009;96:104-110.

7. Schnitzbauer AA, Tsui TY, Kirchner G, et al. Liver transplantation for sclerosing cholangitis in a polytraumtized patient. Nat Clin Pract Gastroenterol Hepatol 2009;6:121-126.

8. de Santibanes E, Ardiles V, Gadano A, Palavecino M, Pekolj J, Ciardullo M. Liver transplantation: the last measure in the treatment of bile duct injuries. Liver Transpl 2008;14:1534-1535.

9. Veroux M, Cillo U, Brolese A, et al. Blunt liver injury: from non-operative management to liver transplantation. Injury 2003;34:181-186.

10. Schroeppel TJ, Croce MA. Diagnosis and management of blunt abdominal solid organ injury. Curr Opin Crit Care 2007;13:399-404.

11. Richardson JD. Changes in the management of injuries to the liver and spleen. J Am Coll Surg 2005;200:648-669.

12. Chiumello D, Gatti S, Caspani L, Savioli M, Fassati R, Gattinoni L. A blunt complex abdominal trauma: total hepatectomy and liver transplantation. Intensive Care Med 2002;28:89-91.

13. Angstadt J, Jarrell B, Moritz M, et al. Surgical management of severe liver trauma: a role for liver transplantation. J Trauma 1989,29:606-608.

14. Polanco P, Leon S, Pineda J, et al. Hepatic resection in the management of complex injury to the liver. J Trauma 2008;65:1269-1270.

15. Tucker ON, Marriott P, Rela M, Heaton N. Emergency liver transplantation following severe liver trauma. Liver Transpl 2008;14:1204-1210.

16. Catalano G, De Simone P, Montin U, et al. Severe liver trauma: the transplant surgeon's perspective. A case report. Hepatogastroenterology 2008;55:1458-1459.

17. Ringe B, Pichelmayr R. Total hepatectomy and liver transplantation: a life-saving procedure in patients with severe hepatic trauma. Br J Surg 1995;82:837-839.

18. Esquivel CO, Bernardos A, Makowka L, Iwatsuki S, Gordon RD, Starzl TE. Liver replacement after massive hepatic trauma. J Trauma 1987;27:800-802.

Received: October 13, 2009 / Accepted: October 19, 2009

Address for correspondence:

Matthias Heuer, M.D.

Department of General-, Visceral- and Transplantation Surgery

University Hospital of Essen

Hufelandstrasse 55

45122 Essen

Germany

Phone: $\quad+49$ (201) 72384010

Fax: $\quad+49(201) 7231137$

E-mail: matthias.heuer@uk-essen.de 